\title{
Feasibility of Mini-Scale Injection Molding for Resource-Efficient Screening of PP-Based Cable Insulation Nanocomposites
}

\author{
Ilkka Rytöluoto ${ }^{1}$, Eetta Saarimäki ${ }^{1}$, Jani Pelto ${ }^{1}$, Mika Paajanen ${ }^{1}$, Xiaozhen $\mathrm{He}^{2}$, Rafal Anyszka $^{2}$, Amirhossein Mahtabani ${ }^{2}$, \\ Wilma Dierkes ${ }^{2}$, Paolo Seri ${ }^{3}$, Hadi Naderiallaf ${ }^{3}$, Kari Lahti $^{4} \&$ Minna Niittymäki ${ }^{4}$ \\ ${ }^{1}$ VTT Technical Research Centre of Finland, Tampere, Finland \\ ${ }^{2}$ University of Twente, Enschede, Netherlands \\ ${ }^{3}$ University of Bologna, Department of Electrical, Electronic and Information Engineering "Guglielmo Marconi", Bologna, Italy \\ ${ }^{4}$ Tampere University, Electrical Engineering, Tampere, Finland
}

\begin{abstract}
This study presents a critical evaluation of the effect of two different sample manufacturing techniques on the morphological and dielectric properties of polypropylene (PP)based nanocomposites, namely mini-scale injection molding (IM) vs. pilot-scale cast film extrusion. Polarized light microscopy revealed that the IM specimen morphology exhibited a layered "skin-core" type morphology, largely differing from the spherulitic morphology of the corresponding extruded cast films. Higher degree of crystallinity in the IM specimens was evidenced by calorimetric and X-ray diffraction methods. The processingdependent morphological differences were found to affect the isothermal charging current (ICC) and thermally stimulated depolarization current (TSDC) characteristics due to differences in charge mobility and trapping, thus making direct comparison of IM and cast film specimens non-straightforward. Nevertheless, mini-scale injection molding can be seen as a resource-efficient sample manufacturing method for facilitating early-stage screening of the best-performing material candidates, given that the morphological features are carefully taken into account.
\end{abstract}

\section{INTRODUCTION}

The engineering dielectric properties of semi-crystalline polymers are known to be closely related with their crystalline structure and bulk morphological features. For instance, the processing-dependent morphology evolution at different scales of structures ranging from small (lamellar) to large (e.g. spherulitic) scale dictates the formation and dielectric properties of the crystalline-amorphous interface [1], thus making processing and specimen preparation non-trivial factors when evaluating and comparing dielectric performance of new materials [2]-[4]. PP based nanocomposite materials are currently being developed for high voltage direct current (HVDC) cable insulation applications in the EU project GRIDABLE, involving material optimization, compounding and sample manufacturing at several different batch sizes at VTT. Recently, mini-scale compounding and injection molding have been identified as a potential resource-efficient alternative for pilot-scale compounding and cast film extrusion to facilitate early-stage screening of the best-performing material candidates. This paper presents a critical comparison of the morphological and dielectric features of PP-based mini-injection molded and extruded cast film specimens, and evaluates the feasibility of the mini-injection molding technique for assessing the effect of nanosilica on the charge trapping and transport properties of PP based nanocomposites.

\section{EXPERIMENTAL}

\section{A. Materials and sample manufacturing}

Various PP-based nanocomposite insulation blends with functionalized nanosilica were prepared at VTT's Polymer Pilot facility. Two main polymer blend systems were utilized in this study: (i) a 55:45 (wt-\%) blend of polypropylene (PP) and poly(ethylene-co-octene) (POE), and (ii) a 55:45 (wt-\%) blend of PP and poly(propylene-ethylene) copolymer (PPE). The polymer blends were mixed with $1.0 \mathrm{wt}-\%$ of surface-functionalized hydrophobic silica nanoparticles labeled herein as "silica A" and "silica B". Moreover, a standard antioxidant package was added to prevent thermo-oxidative degradation. Two main compounding and sample film manufacturing techniques were employed in this study:

- Pilot-scale cast extrusion technique: (i) Large-scale compounding (batch size $1-80 \mathrm{~kg}$ ) by a twin-screw extruder (KraussMaffei Berstorff ZE 25/49D UTX), and (ii) extrusion of the granulated compounds into cast film by a single-screw extruder equipped with a T-die and a calendering system (Brabender Plasticorder).

- Mini-scale injection molding technique: (i) Small-scale compounding (batch size $12 \mathrm{~g}$ ) by a mini-scale twin-screw compounder (Haake MiniLab Rheomex CTW5), and (ii) miniinjection molding (IM) into thin film specimens (Haake MiniJet Pro Piston Injection Moulding System).

For the cast film extrusion the parameters were: temperature profile $195-230{ }^{\circ} \mathrm{C}$, screw speed $85 \mathrm{rpm}$, calendering temperature $80{ }^{\circ} \mathrm{C}$, cast film thickness $300-400 \mu \mathrm{m}$. For the mini-scale compounding and injection molding the parameters were: temperature $230{ }^{\circ} \mathrm{C}$, mixing time $4 \mathrm{~min}$, mixing speed $100 \mathrm{rpm}$, mold temperature $60{ }^{\circ} \mathrm{C}$, pressure $930-1000$ bar, injection and 
hold time $10 \mathrm{~s}+30 \mathrm{~s}$, mold size $26 \times 26 \mathrm{~mm}^{2}$, IM film thickness $\sim 500 \mu \mathrm{m}$.

We remark herein that the focus of the present study is not on the specific nature of the polymer blend or nanosilica surface modification, but rather on comparing the morphological and dielectric features arising from the two different sample manufacturing techniques for a given nanocomposite formulation. Thus, the properties arising from the material itself are only discussed to a limited extent.

\section{B. Morphological characterization}

Differential scanning calorimetry (DSC) measurements were performed for $\sim 5-8 \mathrm{mg}$ disc-shaped cast and IM film specimens encapsulated in aluminium pans using a TA Instruments MDSC2920 (dynamic heating rate of $10^{\circ} \mathrm{C} / \mathrm{min}$, constant nitrogen gas purge of $33 \mathrm{ml} / \mathrm{min}$ ). Polarized light microscopy (POM) was carried out on microtomed sections (thickness $30 \mu \mathrm{m}$ ) by using a Meiji Techno ML8530 microscope equipped with a polarizing facility and a digital camera. Scanning electron microscopy (SEM) was performed on non-coated film specimens broken in liquid nitrogen by using a Zeiss MERLIN HR-SEM scanning electron microscope. Wide angle X-ray diffraction (WAXS) spectra were measured by a Philips X'Pert 1 X-ray diffractometer. The samples were scanned in the $2 \theta$ diffraction angle range of $8-37^{\circ}$ (scanning rate of $0.05 \% / 8 \mathrm{~s}$ ).

\section{Dielectric measurements}

Circular electrodes $(100 \mathrm{~nm} \mathrm{Au})$ were deposited on the both sides of the film specimens by e-beam evaporation. Isothermal charging current (ICC) and thermally stimulated depolarization current (TSDC) measurements were performed using a cryogenic liquid $\left(\mathrm{LN}_{2}\right)$-based temperature control system with an accuracy of $\pm 0.1{ }^{\circ} \mathrm{C}$ (Novocontrol Novocool), a high voltage DC source (Keithley 2290E-5) and an electrometer (Keithley 6517B). A shielded sample cell equipped with a PT100 temperature sensor (Novocontrol BDS1200 HV sample cell) and shielded cables were used. A diode-based overload protection circuit was utilized to protect the electrometer during high voltage application. The consecutive ICC and TSDC measurement procedure was: (i) isothermal $\left(70{ }^{\circ} \mathrm{C}\right)$ polarization at $\mathrm{DC}$ field of $3 \mathrm{kV} / \mathrm{mm}$ for 20 min; measurement of ICC, (ii) rapid cooling to $-50{ }^{\circ} \mathrm{C}$, (iii) removal of the poling voltage and short-circuiting of the sample, (iv) measurement of TSDC under linear heating $\left(3^{\circ} \mathrm{C} / \mathrm{min}\right)$ up to $\sim 145^{\circ} \mathrm{C}$. Space charge measurements were done on selected IM specimens using the pulsed electro-acoustic (PEA) method under $10 \mathrm{kV} / \mathrm{mm}$ DC field (temperature $60{ }^{\circ} \mathrm{C}$ ).

\section{RESULTS AND DISCUSSION}

\section{A. Polarized optical microscopy and SEM}

Exemplifying cross-sectional polarized optical micrographs of microtomed cast film and mini-injection molded (IM) nanocomposite specimens are presented in Figure 1 for both PP/POE and PP/PPE -based blend systems incorporating $1.0 \mathrm{wt}-\%$ of either nanosilica A or B. The cast films (Figure 1a, c \& e) were found to exhibit fine spherulitic morphologies, typical for PP [4], with a mean spherulite size approximated as $<5 \mu \mathrm{m}$ (difficult to detect by OM). The spherulite size in the nanocomposite cast films was found to be much smaller in comparison to the unfilled reference compounds (not shown here). This is due to the nucleating effect of the employed nanosilicas, as discussed elsewhere (see e.g. [5]). On the other hand-in contrast to the extruded cast films-grossly differing cross-sectional morphologies were observed for the corresponding mini-injection molded (IM) specimens (Figure $1 \mathrm{~b}, \mathrm{~d} \& \mathrm{f}$ ): the IM specimen morphologies were found to be of a "skin-core" type which is common for injection molded PP specimens [6]. The outermost layers of the IM films exhibited "skin-layers" (S) with evidence of trans-crystalline growth, being attributable to the nucleating effect and rapid cooling brought by the interior mold surface when the hot polymer melt enters the mold cavity [7]. Beneath the outermost skin-layer where the polymer cooling rate is lower, the presence of an intermediate "shear layer" (SH) and an innermost "core layer" (C) with inhomogeneous (banded) morphologies was confirmed for each IM specimen. The diffused shear and core layers differ in nucleating properties and crystallization rate [8], [9], and are affected by the temperature gradient and pressure during the filling of the mold cavity. While the diffused shear and

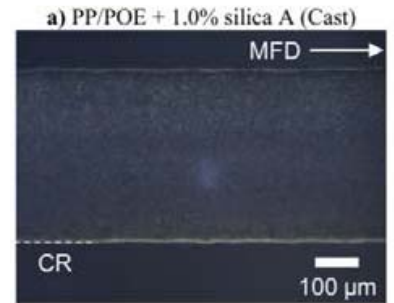

b) $\mathrm{PP} / \mathrm{POE}+1.0 \%$ silica $\mathrm{A}(\mathrm{IM})$

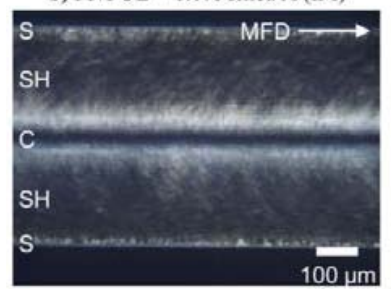

c) $\mathrm{PP} / \mathrm{POE}+1.0 \%$ silica $\mathrm{B}$ (Cast)

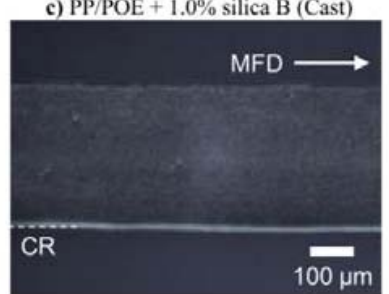

d) $\mathrm{PP} / \mathrm{POE}+1.0 \%$ silica $\mathrm{B}(\mathrm{IM})$

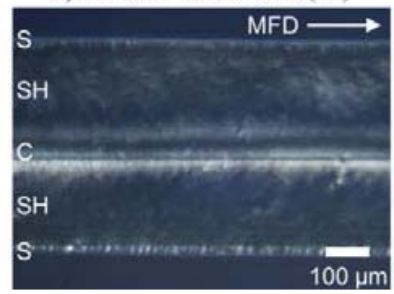

e) PP/PPE $+1.0 \%$ silica B (Cast)

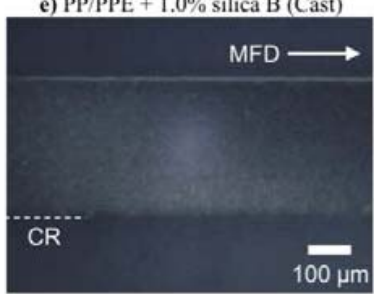

f) $\mathrm{PP} / \mathrm{PPE}+1.0 \%$ silica $\mathrm{B}(\mathrm{IM})$

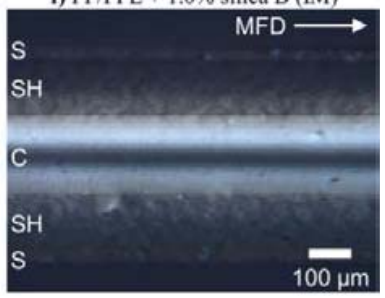

Figure 1. POM cross-sectional images of extruded cast film (top) and mini-injection molded film (bottom) samples. The labeled regions for mini-injection molded (IM) films are S: Trans-crystalline skin-layer, SH: Shear layer, C: Core layer. MFD is the polymer melt flow direction, CR is the chill roll side (cast films). 

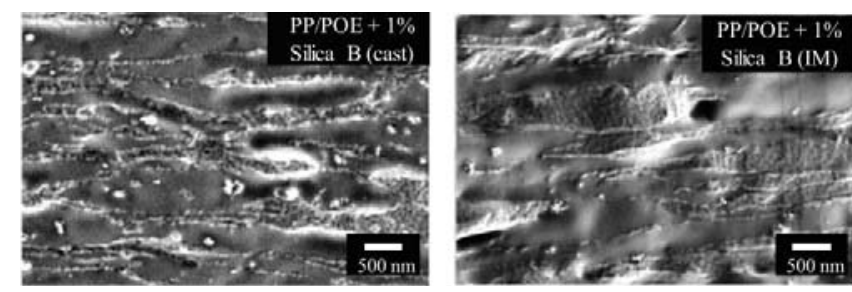

Figure 2. SEM images of PP/POE + 1\% Silica B (left: cast film, right: IM).

core layers may contain spherulites [7], they were too small to be detected by POM, presumably due to a high silica-induced nucleation density in these layers.

It is clear from the POM analysis that significant microstructural differences arise from the studied two sample manufacturing techniques. For the IM samples the oriented skinlayer and the internal shear/core layers can exhibit not only differences in polymer blend morphology and crystallinity but also in filler particle distribution [10], thus having a potentially significant effect on e.g. the trap density and accumulation of injected charge from the electrodes. Furthermore, the presence of distinct layer-like structures within the inner regions of IM specimens can contribute to space charge accumulation due to interfacial polarization, something which is not expected for a smooth spherulitic cast film morphology in similar scale.

Nanoscale silica dispersion in the cast and IM specimens is demonstrated by SEM in Figure 2. In brief, the dispersion and mean particle size were similar for both the methods, indicating no significant differences dispersive mixing. The nanosilica was found to be predominantly located in the PP phase; this effect is discussed in detail elsewhere (see e.g. [5]).

\section{B. Crystallinity (DSC \& WAXS)}

Figure $3 \mathrm{a}-\mathrm{b}$ present DSC $1^{\text {st }}$ heating endotherms of PP/POE and PP/PPE based nanocomposites with 1 wt- $\%$ of silica B manufactured via cast film extrusion and IM techniques, respectively, being representative of the initial specimen morphologies subjected for dielectric measurements. For the PP/POE blend nanocomposites (Figure 3a) two separate melting peaks are observed at $\sim 108{ }^{\circ} \mathrm{C}$ and $\sim 145^{\circ} \mathrm{C}$, corresponding to the melting of the POE and PP phases, respectively, and indicating a bi-phasic (immiscible) blend morphology for PP/POE as has been discussed elsewhere [5]. On the other hand, for the PP/PPE blend nanocomposites (Figure 3b), only a single melting peak of PP phase at $\sim 145{ }^{\circ} \mathrm{C}$ is observed as PP and PPE form a miscible blend. Comparing the DSC peak melting temperatures and enthalpies presented in Figure $3 \mathrm{a}$ and $\mathrm{b}$ for the extruded cast films and IM samples, differences in degree of crystallinity between the two sample manufacturing techniques are revealed, with both the mini-injection molded PP/POE and PP/PPE -based nanocomposites exhibiting slightly higher crystallinities in comparison to their corresponding extruded cast film variants. The observed differences in the DSC crystallinity can be attributed to the differences in micro-morphology, nucleation density and crystallization rate during sample manufacturing as seen in the POM analysis.

The crystalline structure in IM and cast film specimens of $\mathrm{PP} / \mathrm{POE}+1.0 \mathrm{wt}-\%$ silica $\mathrm{B}$ was studied in more detail by WAXS, see Figure 3c. The diffraction peaks at $2 \theta$ angles of 14.1, 16.8, 18.5 , and $25.4^{\circ}$ are characteristic of the $\alpha$-form PP and respectively correspond to (110), (040), (130), and (060) crystallographic planes. The amount of $\beta$-form PP was negligible. In addition to the PP crystallinity, orthorhombic PE crystals corresponding to (110) and (200) crystallographic planes were observed at $2 \theta$ angles of 21.4 and $23.4^{\circ}$, respectively, being attributable to POE. The WAXS spectra confirm the higher degree of crystallinity in the IM specimen, and indicate that this is mainly due to increased crystallinity of the PP phase. Compared with the cast film, the higher intensity of the $\alpha$-form PP (110) and (130) diffraction peaks in the IM specimen can be related to the transcrystalline skin-layer and higher crystalline orientation along the flow direction [7].

\section{Polarization/depolarization characteristics}

Isothermal charging current (ICC) vs. time during the polarization phase (before TSDC measurement) are presented in Figure 4a for selected cast film and IM nanocomposite specimens. As expected, the charging current trends (principally consisting of contributions due to slow polarization, trap filling and conductivity) were decreasing over time. The PP/POE nanocomposite tended to show higher ICC in comparison the PP/PPE based nanocomposites, however the measurement time was far too short to assess the steady-state DC conductivity. Differences in ICC between the extruded cast and IM film specimens were observed, with the IM specimens exhibiting generally lower ICC over time in comparison to their corresponding cast film variants. The lower ICC in the IM samples can be attributed to lower charge mobility caused by the skin-core -type morphology (in particular the presence of transcrystalline skin-layer) and higher crystallinity (i.e. increased amount of crystalline-amorphous interface) which can be postulated to increase the charge trapping propensity.

TSDC spectra of selected samples are presented in Figure
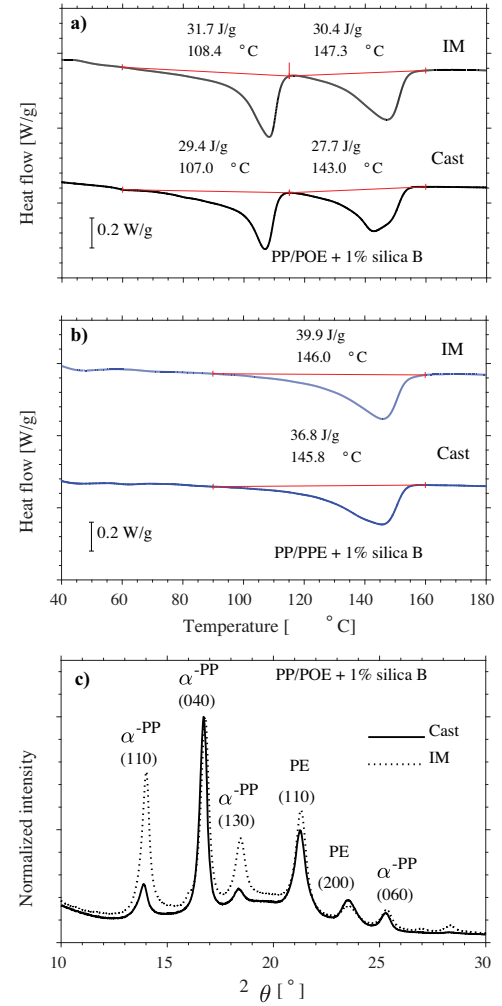

Figure 3. DSC $1^{\text {st }}$ heating endotherms of extruded cast and mini-injection molded (IM) film specimens: a) PP/POE $+1 \%$ silica $\mathrm{B}, \mathrm{b}$ ) $\mathrm{PP} / \mathrm{PPE}+1 \%$ silica $\mathrm{B}$. The melting enthalpies and peak melting temperatures are indicated in the figures. c) WAXS diffraction profiles of extruded PP/POE $+1 \%$ silica $\mathrm{B}$ compounds (cast film and mini-injection molded film). The principal crystalline diffraction peaks of PP and PE phases are labeled. 

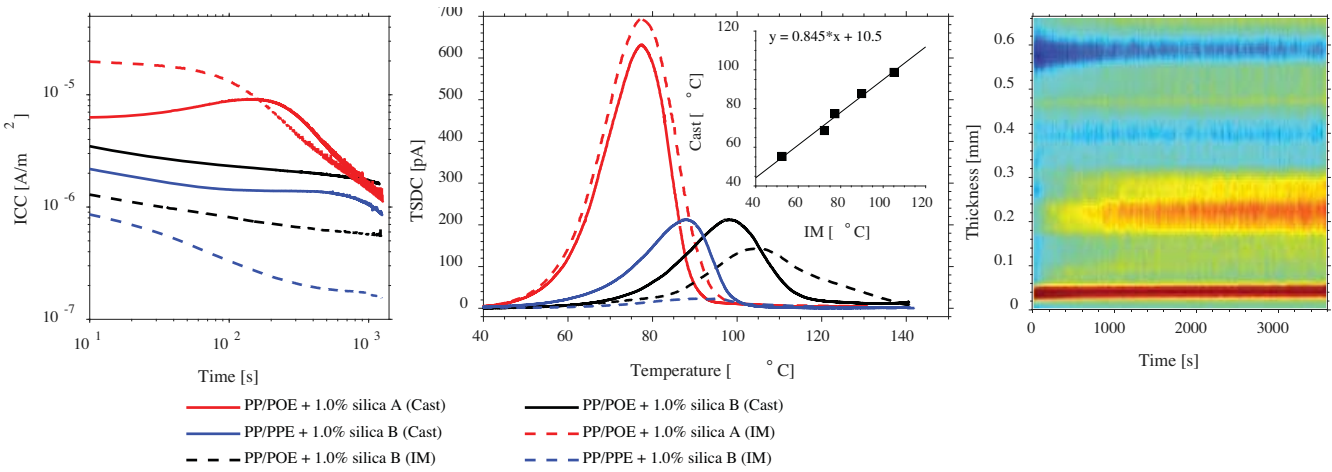

Figure 4. a) Isothermal charging current vs. time (during polarization before TSDC) and b) TSDC spectra for selected extruded cast film and mini-injection molded films. The inset in b) shows the correlation of measured TSDC peak temperature from IM samples (x-axis) with those measured from cast film samples (y-axis) for several different PP/POE and PP/PPE compounds. c) Space charge profile of IM sample $(\mathrm{PP} / \mathrm{POE}+1.0 \mathrm{wt}-\%$ silica B) under $10 \mathrm{kV} / \mathrm{mm}$. IC: Interfacial charge.

$4 \mathrm{~b}$, showing gradual de-trapping of space charge (accumulated during the preceding ICC phase) by thermal stimuli. As an increasing TSDC peak temperature is due to the apparent trap depth being deeper, the nanocomposites with silica B were found to contain deeper traps than those filled with silica A. The TSDC peak intensity and the integrated area (released charge) are related with the apparent density of traps filled during the polarization, and they were correlated with the integrated charge calculated from the preceding isothermal polarization curves. Comparing the TSDC of extruded cast and IM film specimens, significant differences arising from the sample manufacturing method and morphology were observed. The IM samples which showed lower ICC (during poling) also showed lower TSDC peak intensity. The inset in Figure $4 \mathrm{~b}$ compares the TSDC peak temperatures determined from the IM samples (x-axis) against those from cast film samples (y-axis): these were correlated, however the IM samples tended to show slightly higher peak temperatures (trap depths) in comparison to cast films. Nevertheless, the trends were always systematic for both IM and cast films, i.e. the effect of nanosilica on the trap depth was relatively similar for both sample types. Lastly, Figure $4 \mathrm{c}$ presents an exemplifying PEA space charge profile of a PP/POE-silica B IM specimen: the interfacial charge build-up due to the layered skin-core -type morphology is evident, highlighting the morphological differences which can arise from the sample preparation method.

\section{CONCLUSIONS}

The layered "skin-core" -type morphology of mini-injection molded (IM) specimens is significantly different from the spherulitic morphology of extruded cast films, and this has profound effects on charge injection, transport and trapping under high DC electric field. On the other hand, the effect of nanosilica on the apparent charge trap depth is consistent for the both sample manufacturing methods. Therefore, mini-scale injection molding can be seen as a resource-efficient alternative for pilot-scale compounding and cast film extrusion for facilitating early-stage screening of the best-performing material candidates, given that the morphological dissimilarities are carefully taken into account.

\section{ACKNOWLEDGMENT}

This project has received funding from the European Union's Horizon 2020 research and innovation programme under grant agreement No 720858 .

\section{REFERENCES}

[1] R. Bartnikas and R. Eichhorn, Engineering Dielectrics Volume IIA Electrical Properties of Solid Insulating Materials: Molecular Structure and Electrical Behavior. Philadelphia: ASTM International, 1983.

[2] I. L. Hosier, A. S. Vaughan, and S. G. Swingler, "The effects of measuring technique and sample preparation on the breakdown strength of polyethlyene," IEEE Trans. Dielectr. Electr. Insul., vol. 9, no. 3, pp. 353-361, 2002.

[3] M. Karlsson, X. Xu, K. Gaska, H. Hillborg, S. Gubanski, and U. W. Gedde, "DC Conductivity Measurements of LDPE: Influence of Specimen Preparation Method and Polymer Morphology," Proc. Nord. Insul. Symp., Oct. 2017.

[4] I. Rytöluoto, A. Gitsas, S. Pasanen, and K. Lahti, "Effect of film structure and morphology on the dielectric breakdown characteristics of cast and biaxially oriented polypropylene films," Eur. Polym. J., vol. 95, 2017.

[5] X. He et al., "Surface modification of fumed silica by plasma polymerization of acetylene for $\mathrm{PP} / \mathrm{POE}$ blends dielectric nanocomposites," Polymers (Basel)., vol. 11, no. 12, 2019.

[6] M. Fujiyama, Y. Kitajima, and H. Inata, "Structure and properties of injection-molded polypropylenes with different molecular weight distribution and tacticity characteristics," J. Appl. Polym. Sci., vol. 84, no. 12, pp. 2142-2156, 2002.

[7] P. W. Zhu and G. Edward, "Studies of injection-moulded isotactic poly(propylene) by synchrotron WAXD/SAXS: Effects of nucleating agent on morphological distribution," Macromol. Mater. Eng., vol. 288, no. 4, pp. 301-311, 2003

[8] M. R. Kantz, H. D. Newman, and F. H. Stigale, "The skin-core morphology and structure-property relationships in injection-molded polypropylene," J. Appl. Polym. Sci., vol. 16, no. 5, pp. 1249-1260, 1972.

[9] P. Deng, B. Whiteside, F. Wang, K. Norris, and J. Zhang, "Epitaxial growth and morphological characteristics of isotactic polypropylene/polyethylene blends: Scale effect and mold temperature," Polym. Test., vol. 34, pp. 192-201, 2014.

[10] J. Karger-Kocsis and I. Csikai, "Skin-Core morphology and failure of injection-molded specimens of impact-modified polypropylene blends," Polym. Eng. Sci., vol. 27, no. 4, pp. 241-253, Feb. 1987. 